\title{
Thyroid stimulating autoantibody M22 mimics TSH binding to the TSH receptor leucine rich domain: a comparative structural study of protein-protein interactions
}

\author{
R Núñez Miguel ${ }^{1,2}$, J Sanders ${ }^{1}$, D Y Chirgadze ${ }^{2}$, J Furmaniak ${ }^{1}$ and B Rees Smith ${ }^{1}$ \\ ${ }^{1}$ FIRS Laboratories, RSR Ltd, Parc Ty Glas, Llanishen, Cardiff CF14 5DU, UK \\ ${ }^{2}$ Department of Biochemistry, University of Cambridge, 80 Tennis Court Road, Cambridge CB2 1GA, UK \\ (Correspondence should be addressed to B Rees Smith; Email: firs@ rsrltd.eclipse.co.uk)
}

\begin{abstract}
The TSH receptor (TSHR) ligands M22 (a thyroid stimulating human monoclonal antibody) and TSH, bind to the concave surface of the leucine rich repeats domain (LRD) of the TSHR and here, we show that M22 mimics closely the binding of TSH. We compared interactions produced by M22 with the TSHR in the M22-TSHR crystal structure (2.55 A resolution) and produced by TSH with the TSHR in a TSH-TSHR comparative model. The crystal structure of the TSHR and a comparative model of TSH based on the crystal structure of FSH were used as components to build the TSH-TSHR model. This model was built based on the FSH-FSH receptor structure $(2.9 \AA)$ and then the structure of the TSHR in the model was replaced by the TSHR crystal structure. The analysis shows that M22 light chain mimics the TSH $\beta$ chain in its interaction with TSHR LRD, while M22 heavy chain mimics the interactions of the TSH $\alpha$ chain. The M22-TSHR complex contains a greater number of hydrogen bonds and salt bridges and fewer hydrophobic interactions than the TSH-TSHR complex, consistent with a higher M22 binding affinity. Furthermore, the surface area formed by TSHR residues N208, Q235, R255, and N256 has been identified as a candidate target region for small molecules which might selectively block binding of autoantibodies to the TSHR.
\end{abstract}

Journal of Molecular Endocrinology (2009) 42, 381-395

\section{Introduction}

Detailed studies of molecular mimicry (Wucherpfennig 2001) provide insights into the nature of protein interactions that mediate key signalling pathways involved in cell regulation. The phenomenon of molecular mimicry is found in antibodies and as an example, two antibodies, specific for the activated conformation of the adhesion receptor integrin $\alpha \mathrm{v} \beta 3$ that is associated with a metastatic phenotype, were found to contain the Arg-Gly-Asp integrin recognition motif of the natural ligand within the third complementarity-determining region of the heavy chain (HC; Felding-Habermann et al. 2004). Of particular significance are antibodies that are able to mimic hormone-receptor interactions in such a way as to give rise to full activation of receptor mediated signal transduction. Here, we report a detailed analysis of interactions involving an autoantibody that activates the TSH receptor (TSHR) in Graves' disease (Sanders et al. 2003, 2007a, Rees Smith et al. 2007) and a comparison of these interactions with those of TSH with the TSHR.

TSH belongs to the glycoprotein hormone (GPH) family and binds to the TSHR on the surface of thyroid follicular cells (Rees Smith et al. 1988, Grossmann et al. 1997). The hormone regulates production and secretion of the thyroid hormones thyroxine and triiodothyronine via a negative feedback system involving a hypothalamic-pituitary-thyroid axis (Smith $e$ al. 2002, Szkudlinski et al. 2002). The two subunits ( $\alpha$ and $\beta)$ of TSH interact non-covalently and are $\mathrm{N}$-glycosylated. Glycosylation is important for proper folding, assembly, secretion, and biological activity of TSH (Baenziger \& Green 1988, Grossmann et al. 1995, Szkudlinski et al. 1996). A cystine-knot motif is found in the structure of each TSH subunit (Murray-Rust et al. 1993). There are five intra chain disulphide bonds in the $\alpha$-subunit and six in the $\beta$-subunit (McDonald \& Hendrickson 1993). The cystine-knot core contains two disulphide bonds between adjacent antiparallel strands forming a ring which is penetrated by a third disulphide bond. From the knot, a loop of double-stranded $\beta$-sheet-like structure can be observed on one side while on the other side there are two almost parallel hairpin loops (Wu et al. 1994, Fox et al. 2001).

The TSHR belongs to the rhodopsin-like class of G protein-coupled receptors (GPCRs; Fredriksson et al. 2003, Foord et al. 2005) and consists of an extracellular region formed by a TSHR domain consisting of

DOI: 10.1677/JME-08-0152 Online version via http://www.endocrinology-journals.org 
ten leucine-rich repeats (leucine rich domain; LRD) with its N-terminal end linked by S-S bonds (Sanders et al. 2007a) and a cleavage domain (CD) containing six half-cystines; part of the $\mathrm{CD}$ can be cleaved out during receptor processing and/or degradation (Rees Smith et al. 1988, Chazenbalk et al. 1996, Rapoport et al. 1998, Núñez Miguel et al. 2003). The transmembrane domain (TMD) is predicted to have seven membrane-spanning helices similar to those of rhodopsin and the structure is completed by a cytoplasmic tail (Szkudlinski et al. 2002, Farid \& Szkudlinski 2004, Núñez Miguel et al. 2004). TSH binding to the TSHR initiates an activation cascade with the activation signal being transduced through the TMD causing activation of cytoplasmic heterotrimeric $\mathrm{G}_{\mathrm{s}}$ and $\mathrm{G}_{\mathrm{q}}$ proteins (Claus et al. 2006). To date, the crystal structure of several GPCRs, with short extracellular regions, have been solved i.e. rhodopsin (Palczewski et al. 2000, Murakami \& Kouyama 2008), opsin (Scheerer et al. 2008), $\beta_{2}$-adrenergic receptor (Cherezov et al. 2007, Rasmussen et al. 2007), $\beta_{1}$ adrenergic receptor (Warne et al. 2008), and $A_{2 A}$ adenosine receptor (Jaakola et al. 2008). Out of the GPH receptors characterized by large extracellular domains, the crystal structure of a part of the TSHR LRD in complex with M22 has been solved at $2.55 \AA$ resolution (Sanders et al. 2007a) and the $2.9 \AA$ resolution structure of a part of the FSH receptor (FSHR) in complex with FSH has been reported (Fan \& Hendrickson 2005).

In Graves' disease autoantibodies to the TSHR mimic the actions of TSH and cause thyroid overactivity characterized by high serum thyroid hormone levels and low serum TSH levels. The clinical symptoms also include Graves' ophthalmopathy (Rees Smith et al. 1988, 2007, Sanders et al. 2002). M22 is a human monoclonal autoantibody to the TSHR prepared using lymphocytes from a patient with Graves' disease. It has powerful thyroid stimulating and TSH binding inhibiting activity (Sanders et al. 2003, 2004) and binds to the TSHR with high affinity $\left(5 \times 10^{10} \mathrm{l} / \mathrm{mol}\right.$; Sanders et al. 2003, 2004). The unbound M22 Fab crystal structure, solved at 1.65 Å resolution (Sanders et al. 2004), shows a typical Fab-fragment structure but with an unusual relative position between the variable regions of the $\mathrm{HC}$ and the light chain (LC). It is of interest that the structure of M22 bound to the TSHR determined at $2 \cdot 55 \AA$ resolution showed virtually no movement in the atoms of M22 residues compared with unbound M22 (Sanders et al. 2007a).

Here, we describe a comparative analysis of the interactions between the TSHR and TSH and between the TSHR and M22. The TSHR-M22 Fab-crystal structure (Sanders et al. 2007a) and predictions from a comparative model of the TSH-TSHR complex (Núñez Miguel et al. 2008) were analysed in the light of experimental evidence on receptor residues that are important for TSH and M22 binding and biological activity. The study shows that M22 mimics closely binding of TSH to the TSHR. Furthermore, M22 LC interactions mimic those of TSH $\beta$, whereas M22 $\mathrm{HC}$ interactions mimic those of TSH $\alpha$. Comparison of interactions in the M22-TSHR and TSH-TSHR complexes indicate that they are consistent with the observed high affinity of the M22-TSHR interaction i.e. higher than TSH-TSHR interaction. Furthermore, we have identified an area on the surface of the TSHR LRD which is a candidate target for small-molecule therapeutic agents with potential to block binding of TSHR autoantibodies.

\section{Methods}

Analyses of interactions were carried out using the 2.55 ̊ resolution M22-TSHR LRD structure (Sanders et al. 2007a, 2008a, Protein Data Bank ID: 3G04) and the model of the TSH-TSHR LRD complex (Núñez Miguel et al. 2008). In the absence of an experimental structure, a comparative model of the TSH-TSHR LRD complex (Fig. 1A) based on the crystal structures of the FSH-FSHR LRD at $2 \cdot 9$ A resolution (Fan \& Hendrickson 2005) and the M22-TSHR LRD at $2.55 \AA$ resolution (Sanders et al. 2007a) described before (Núñez Miguel et al. 2008) was used in our study. This model (Fig. 1A) showed good geometric and conformational parameters and has been validated further using evidence obtained from experimental mutations (Núñez Miguel et al. 2008).

Van der Waals interactions between residues and shape complementarity in the interfaces of the complexes were studied using accessible surface areas (ASA), calculated using the Lee \& Richards algorithm (Lee \& Richards 1971) developed by Richmond (1984). The gap volume between two interacting proteins was used to study the complementarity of the interacting surfaces. The gap volume was calculated using the program SURFNET (Laskowski 1995). The gap volume index is calculated using the formula: Gap Volume Index $=$ Gap Volume/Interface ASA ( Jones \& Thornton 1996). Hydrogen bonds were defined by the program HBPLUS (McDonald \& Thornton 1994). The program defines a hydrogen bond when the distances between donor and acceptor atoms are lower than or equal to $3.5 \AA$ and the angles between donor-hydrogen-acceptor and donor-acceptor-acceptor adjacent are greater or equal to $90^{\circ}$. Electrostatic interactions between atoms of charged residues were obtained using an in house program (ELECINT, Núñez Miguel unpublished observations). ELECINT obtains atomic charges by using the Henderson-Hasselbalch equation and applies a distance-dependent dielectric constant. 

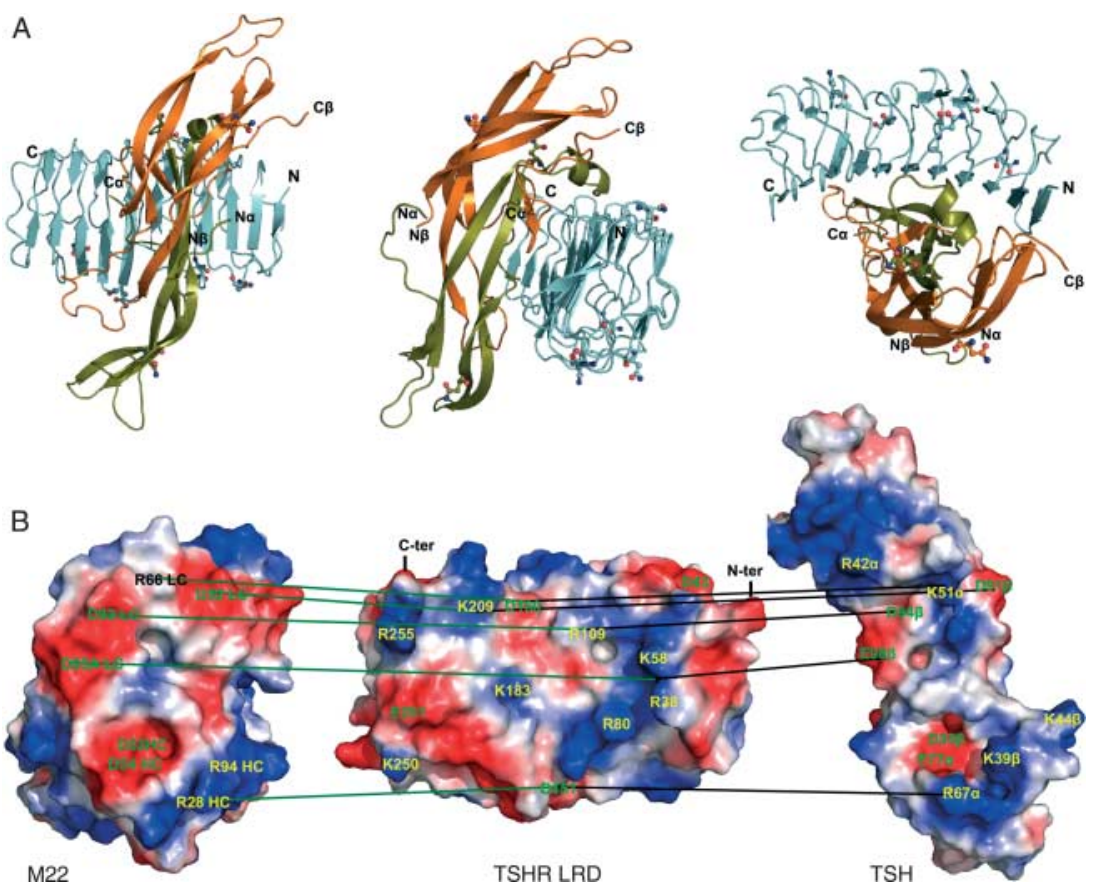

Figure 1 Model of the TSH-TSHR complex (Núñez Miguel et al. 2008). (A) Three orthogonal views of the TSH-TSHR LRD complex. The LRD of the TSHR is shown in cyan, the $\alpha$-chain of the TSH in olive and the $\beta$-chain in orange. $\mathrm{N}$ - and $\mathrm{C}$-termini of all chains are marked. TSH and TSHR arginines that are $\mathrm{N}$-glycosylated are shown as ball and stick with the carbon atoms in cyan (TSHR), olive (TSH $\alpha$ ), and orange (TSH $\beta)$, oxygens in red and nitrogens in blue. (B) Electrostatic potential of the interacting surfaces of M22, TSHR LRD, and TSH showing some important charged residues. The $\mathrm{N}$ - and $\mathrm{C}$ - termini of TSHR LRDs are marked. Some important interactions between the receptor and the ligands are shown by connecting lines (see text for details). Negative patches are shown in red and positive in blue.

\section{Results}

\section{M22-TSHR and TSH-TSHR interfaces}

Twelve M22 LC residues and 16 M22 HC residues gave rise to interactions $>10 \AA^{2} \Delta \mathrm{ASA}$ (difference between ASA values before and after complexation) on complexation with 28 TSHR residues. Fifteen TSH $\alpha$-chain residues and 14 TSH $\beta$ chain residues produced interactions $>10 \AA^{2} \Delta$ ASA on complexation with 31 TSHR residues. All M22 and TSH residues that are closer than $4 \AA$ to TSHR residues and those at a distance between 4 and $7 \AA$ having electrostatic interactions are shown in Supplementary Table 1, see supplementary data in the online version of the Journal of Molecular Endocrinology at http://jme.endocrinology-journals. org/content/vol42/issue5/. Several important similarities and differences can be observed in the interfaces of the M22-TSHR and TSH-TSHR complexes. Table 1 gives the numbers of TSH residues that interact with each repeat of the TSHR and Table 2 shows the number and type of interactions in the interfaces of the two complexes. The M22-TSHR complex has more salt bridges $(n=8)$ and hydrogen

Table 1 Number of residues from M22 (light and heavy chains) and TSH ( $\alpha$ and $\beta$-chains) that interact with residues of TSH receptor (TSHR) leucine rich repeats

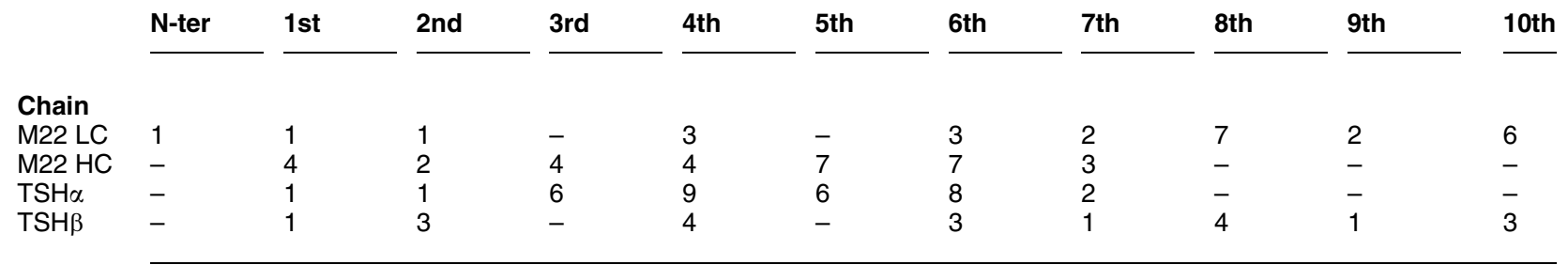


Table 2 Number and type of interactions in the interfaces of the M22-TSH receptor (TSHR) and TSH-TSHR complexes

\begin{tabular}{|c|c|c|c|c|c|}
\hline Salt bridges & $\begin{array}{l}\text { Hydrogen } \\
\text { bonds }\end{array}$ & Ion pairs & Dipole-dipole & $\begin{array}{l}\text { Dipole-induced } \\
\text { dipole }\end{array}$ & Hydrophobic \\
\hline 8 & 14 & 10 & 17 & 36 & 14 \\
\hline 4 & 3 & 8 & 18 & 36 & 22 \\
\hline
\end{tabular}

bonds $(n=14)$, and fewer hydrophobic interactions $(n=14)$ compared with the TSH-TSHR complex ( $n=4 ; n=3$ and $n=22$ of salt bridges, hydrogen bonds and hydrophobic interactions respectively).

Although there is no strict quantitative relationship between interface areas of a complex or shape complementarity of interfaces, these parameters do give an indication of the extent of the interactions. Table 3 shows that the interface $\triangle$ ASAs are similar in both complexes with $\triangle$ ASA in the TSH-TSHR complex $\left(2533 \AA^{2}\right.$ ) only being $19 \AA^{2}$ greater than the M22-TSHR complex $\left(2514 \AA^{2}\right)$. However, the gap volume index of the TSH-TSHR complex is $26 \%$ higher than that of the M22-TSHR complex (3.66 vs 2.91 respectively). We have previously found that the gap volume index calculations for theoretical models are fairly robust and not too dependent on the accuracy of the models (Núñez Miguel et al. 2008).

Electrostatic potential surfaces of the interacting faces of M22 and TSH with TSHR LRD (Fig. 1B) indicate some similarities between M22 and TSH. The area on the TSH surface involving residues D35 $\beta$, E77 $\alpha, \mathrm{K} 39 \beta$ and R67 $\alpha$ is similar to the M22 surface area involving residues D52 HC, D54 HC, R94 HC, and R28 HC. Furthermore, electrostatic potential surface area of TSH formed by residues D91 $\beta$, K51 $\alpha$, D94 $\beta$, and E98 $\beta$ is similar to the M22 area formed by residues D52 LC, R66 LC, D93 LC, and D95A LC. However, the electrostatic potential of M22 R66 LC is markedly reduced (pale blue in Fig. 1B) by an intramolecular saltbridge with M22 D51 LC.

Table $3 \Delta$ Accessible surface areas ( $\triangle \mathrm{ASA})$, gap volumes, and gap volume indexes in the interfaces of the M22-TSH receptor (TSHR) and TSH-TSHR complexes

\begin{tabular}{|c|c|c|c|}
\hline & $\begin{array}{l}\text { Interface } \\
\text { ASA }\left(\AA^{2}\right)\end{array}$ & $\begin{array}{l}\text { Gap } \\
\text { volume }\left(\AA^{3}\right)\end{array}$ & $\begin{array}{l}\text { Gap volume } \\
\text { index }(\AA)\end{array}$ \\
\hline M22 & 1257 & & \\
\hline TSHR & 1257 & & \\
\hline M22-TSHR & 2514 & 7308 & $2 \cdot 91$ \\
\hline TSH & 1292 & & \\
\hline TSHR & 1241 & & \\
\hline TSH-TSHR & 2533 & 9279 & 3.66 \\
\hline
\end{tabular}

\section{Interface residue interactions}

Hydrogen bonds, van der Waals interactions and charged electrostatic interactions observed in the interfaces of the M22-TSHR LRD and TSH-TSHR LRD were studied. In our analyses, the maximum distance considered for hydrogen bonds was $3.5 \AA$, for van der Waals interactions $4 \AA$ and for electrostatic interactions $7 \AA$. In the study of charge-charge interactions, a 'salt bridge' was considered when the atomic distance between the two charged atoms was less than or equal to $3.5 \AA$, an 'ion pair' was considered when the atomic distance was $>3.5 \AA$ and less than or equal to $4.5 \AA$, and those at the distance longer than $4.5 \AA$ were considered to be 'solvent-separated ion pairs'. Supplementary Table 1 shows that in the M22TSHR and TSH-TSHR complexes, 26 TSHR residues are involved in interactions with M22, 30 TSHR residues interact with TSH, 10 TSHR residues that interact with M22 do not interact with TSH, and 14 TSHR residues that interact with TSH do not interact with M22.

\section{Similar interactions in the two complexes}

Although the two TSHR ligands (M22 and TSH) have different structures, we found several interactions between TSHR residues and M22 and TSH residues that are similar in the two complexes, i.e. similar types of interaction produced by the same or similar amino acids from the ligand in equivalent positions in the space with the same receptor residue. Table 4 shows those residues and their interactions.

TSHR K58 forms a salt bridge with M22 LC D95A and a hydrogen bond with M22 HC Y99, while in the TSHTSHR complex, TSHR K58 forms an ion pair with E98 from TSH $\beta$ (Table 4, Fig. 2A). In addition, TSH $\beta$ E98 forms also a solvent-separated ion pair with TSHR R38 (Table 5). All these residues are exposed to the solvent.

TSHR mutation K58A would be expected to disrupt the ion pair between TSHR K58 and TSH $\beta$ E98 but the solvent-separated ion pair between TSHR R38 and TSH $\beta$ E98 should remain intact (Table 5). Consequently, the TSHR K58A mutation would not be expected to have an impact on TSH binding affinity to the TSHR and this is observed in mutation experiments (Sanders et al. 2006). M22 HC Y99 is 
Table 4 Comparison of interactions in the M22-TSH receptor (TSHR) and TSH-TSHR complexes - similar interactions produced by TSHR residues

\begin{tabular}{|c|c|c|c|c|c|c|c|}
\hline & Main interaction & M22 & TSHR & TSHR & TSH & Main interaction & Distance $(\AA)$ \\
\hline \multicolumn{8}{|c|}{ Distance $(\AA)$} \\
\hline $2 \cdot 6$ & Salt bridge & $D 95 A L C$ & K58 & K58 & $E 98 \beta$ & Ion pair & $4 \cdot 0$ \\
\hline $3 \cdot 7$ & Hydrophobic & P97 HC & F130 & F130 & $Y 88 \alpha$ & Hydrophobic & $3 \cdot 5$ \\
\hline $3 \cdot 8$ & Hydrophobic & G98 HC & & & & & \\
\hline $2 \cdot 8$ & Salt bridge & $R 28 H C$ & D151 & D151 & $R 67 \alpha$ & Solvent-separated ion pair & $6 \cdot 8$ \\
\hline $3 \cdot 8$ & Cation- $\pi$ & $R 28 H C$ & & & $K 39 \beta$ & Cation- $\pi$ & $2 \cdot 9$ \\
\hline $3 \cdot 5$ & Polar & S31 HC & & & & & \\
\hline $3 \cdot 7$ & Hydrophobic & Y32 HC & $\mid 155$ & $\mid 155$ & $Y 89 \alpha$ & Hydrophobic & $2 \cdot 8$ \\
\hline 3.9 & Hydrophobic & P97 HC & & & & & \\
\hline $6 \cdot 7$ & Unfavourable electrostatic & $D 51 L C$ & D160 & D160 & $D 94 \beta$ & Unfavourable electrostatic & $6 \cdot 6$ \\
\hline $5 \cdot 1$ & Solvent-separated ion pair & $R 66 L C$ & & & $K 91 \alpha$ & Solvent-separated ion pair & $5 \cdot 1$ \\
\hline $3 \cdot 6$ & Ion pair & $D 52 L C$ & K209 & K209 & $D 91 \beta$ & Salt bridge & $2 \cdot 7$ \\
\hline $3 \cdot 5$ & Polar & Y50 LC & & & & & \\
\hline $4 \cdot 5$ & Ion pair & D51 LC & & & & & \\
\hline $3 \cdot 3$ & Induced dipole & Q53 LC & & & & & \\
\hline $6 \cdot 4$ & Solvent-separated ion pair & R66 LC & & & & & \\
\hline
\end{tabular}

Ligand residues producing similar interactions are shown in italics. Ion-dipole and dipole-dipole interactions not involved in hydrogen bonds are described as 'polar'. Ion-induced dipole and dipole-induced dipole interactions are described as 'induced dipole'. Dispersion and hydrophobic forces are described as 'hydrophobic'. Charge-charge interactions at distance $\leq 3.5 \AA$ are defined as 'salt bridges'. Charge-charge interactions at distance $>3.5$ and $\leq 4.5 \AA$ are defined as 'ion pairs'. Charge-charge interactions at $>4.5 \AA$ distance are defined as 'solvent-separated ion pairs'. For explanation of cation- $\pi$ interactions see Gallivan \& Dougherty (1999).

hydrogen bonded with TSHR residues K58 and E107 (Table 6). Disruption of one of these hydrogen bonds should not have an effect on M22 binding and activity and this is observed in mutation studies involving TSHR K58 (Sanders et al. 2006).

Hydrophobic interactions are observed between the TSHR residue F130 and M22 HC P97 in the M22-TSHR complex and TSH $\alpha$ Y88 in the TSH-TSHR complex (Table 4, Fig. 2B). However, the TSHR F130A mutant showed a reduced binding affinity for M22 but not for porcine TSH (Sanders et al. 2006) and this suggests that the interaction between TSHR F130 and M22HC P97 is likely to be stronger than the interaction between the TSHR F130 and TSH $\alpha$ Y88. It is of interest that M22HC $\mathrm{P} 97$ is the only residue with a position in the complex that deviates $(1 \cdot 1 \AA)$ from its position in unbound M22 (Sanders et al. 2007a). Furthermore, P97 is one of the HC CDR3 residues that have undergone mutation from the germ line gene sequence (Sanders et al. 2004) and this may be related to the importance of HC P97 in the interactions between M22 and the TSHR.

There are charge-charge interactions between TSHR D151 and M22HC R28 in the M22-TSHR complex and TSH $\alpha$ R67 in the TSH-TSHR complex (Table 4, Fig. 2C). In both cases, the charges of the interacting residues are exposed to the solvent. Reduction of M22 stimulated cyclic AMP production to $60-80 \%$ of the wild-type activity has been reported for the M22 HC R28D mutation (Sanders et al. 2007b) and this is consistent with the importance of the charge-charge interaction involving M22HC R28 even when exposed to solvent and irrespective of M22HC R28 producing other minor interactions with the TSHR (Tables 4 and 6 and see below).

TSHR I152 gives rise to hydrophobic interactions with the aliphatic part of the side chains of M22HC R28 as well as TSH $\alpha$ R67 in the respective complexes and close contacts with the charged atoms of both arginines (Table 4, Fig. 3A).

TSHR F153 and I155 interact in a similar way with M22HC Y32 and R28 and with TSH $\alpha$ residues Y89 and TSH $\beta$ K39 in the respective complexes. In both cases, the hydroxyl groups of the interacting tyrosines are facing the respective TSHR residues and the charged regions of the ligands are close to the aromatic ring of TSHR F153 in a cation- $\pi$ interaction (Gallivan \& Dougherty 1999; Table 4, Fig. 3B). The M22 HC R28D mutant has reduced stimulating activity $(60-80 \%$ of the wild-type; Sanders et $a l$. 2007b) in accordance with an absence of the cation $-\pi$ interaction in the mutant. 

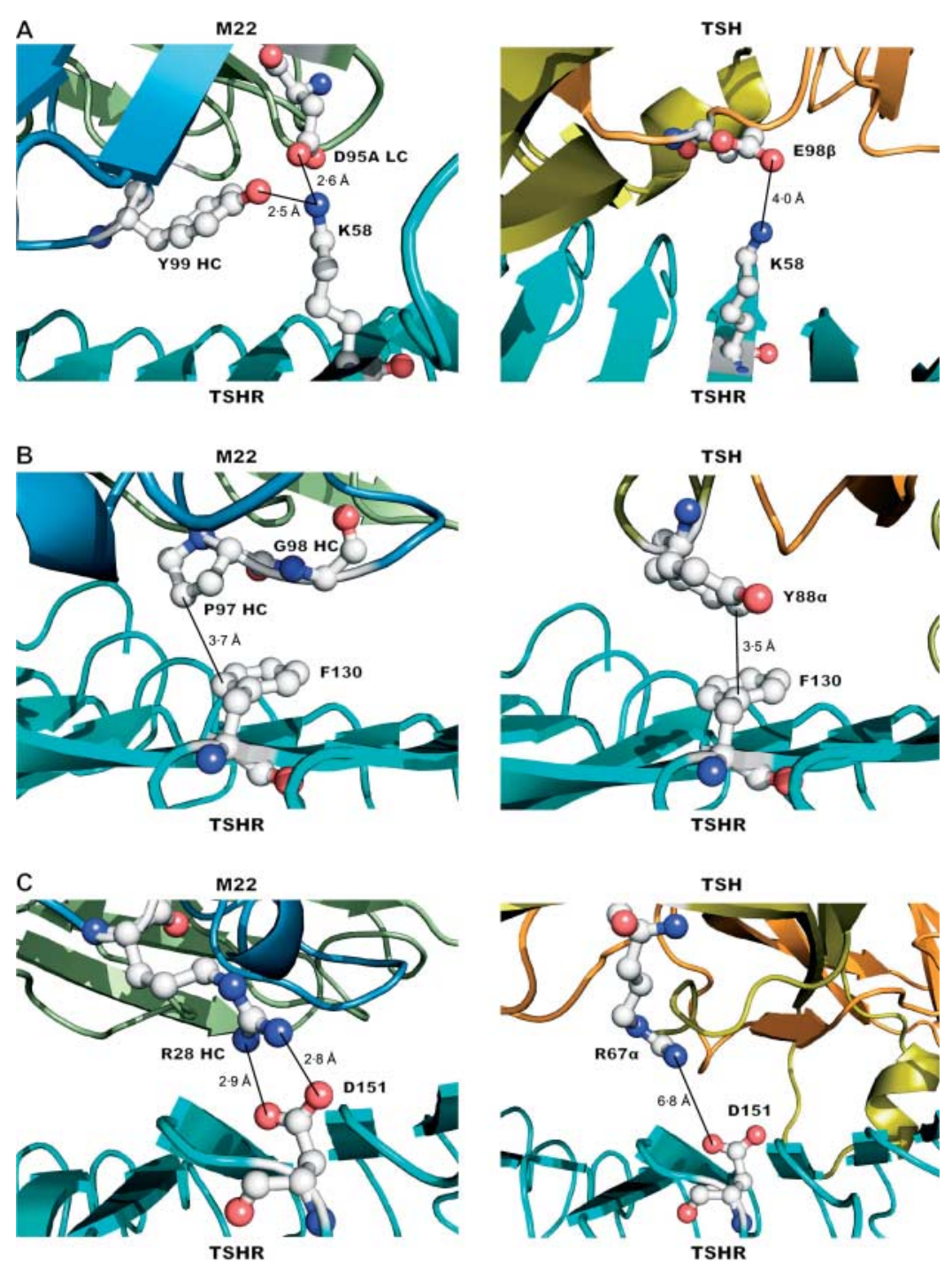

Figure 2 Interactions produced by (A) TSHR K58 with M22 residues and with TSH $\beta$ E98, (B) TSHR F130 with M22 heavy chain residues and with $\mathrm{TSH} \alpha \mathrm{Y} 88$, and (C) TSHR D151 with M22 heavy chain R28 and with $\mathrm{TSH} \alpha \mathrm{R} 67$ in the M22-TSHR and TSHTSHR complexes. TSHR LRD backbones are shown in cyan, hormone $\alpha$-chains in olive and $\beta$-chains in orange, M22 light chain in green and heavy chain in blue. Interacting residues are shown as ball and sticks with carbon atoms in grey, oxygens in red, and nitrogens in blue. Solid lines denote interactions.

TSHR D160 is involved in both favourable and unfavourable charge-charge interactions with M22 residues; an ion-pair with M22 LC R66 and an unfavourable charge-charge interaction with M22LC D51. Similarly, TSHR D160 interacts with TSH $\alpha$ K91 and TSH $\beta$ D94 in the TSH-TSHR complex. In the latter complex, TSHR D160 is involved in additional interactions with $\mathrm{TSH} \alpha$ and $\mathrm{TSH} \beta$ residues as shown in Fig. 3C and Table 4.

A similar reduction in the ability to simulate the TSHR D160A mutant is observed for TSH and for M22 (60-80\% of the wild-type in both cases; Sanders et al.
$2007 b$ ). This reduction may be due to disruption of charge-charge interactions (Table 4). However, the reduction of stimulating activity in the case of TSHR D160K mutation is greater for TSH $(<20 \%$ of the wildtype) than for M22 (80-100\% of the wild-type; Sanders et al. 2007b). It can be predicted that in the TSH-TSHR complex, the D160K mutation would produce one unfavourable charge-charge interaction more (with TSH $\alpha$ K51) than in the M22-TSHR complex (Fig. 3C).

The positively charged amino group of TSHR K183 makes a cation- $\pi$ interaction with the aromatic ring of M22 HC W100C and similarly TSHR K183 interacts with 
Table 5 Comparison of interactions in the M22-TSH receptor (TSHR) and TSH-TSHR complexes - different interactions produced by TSHR residues

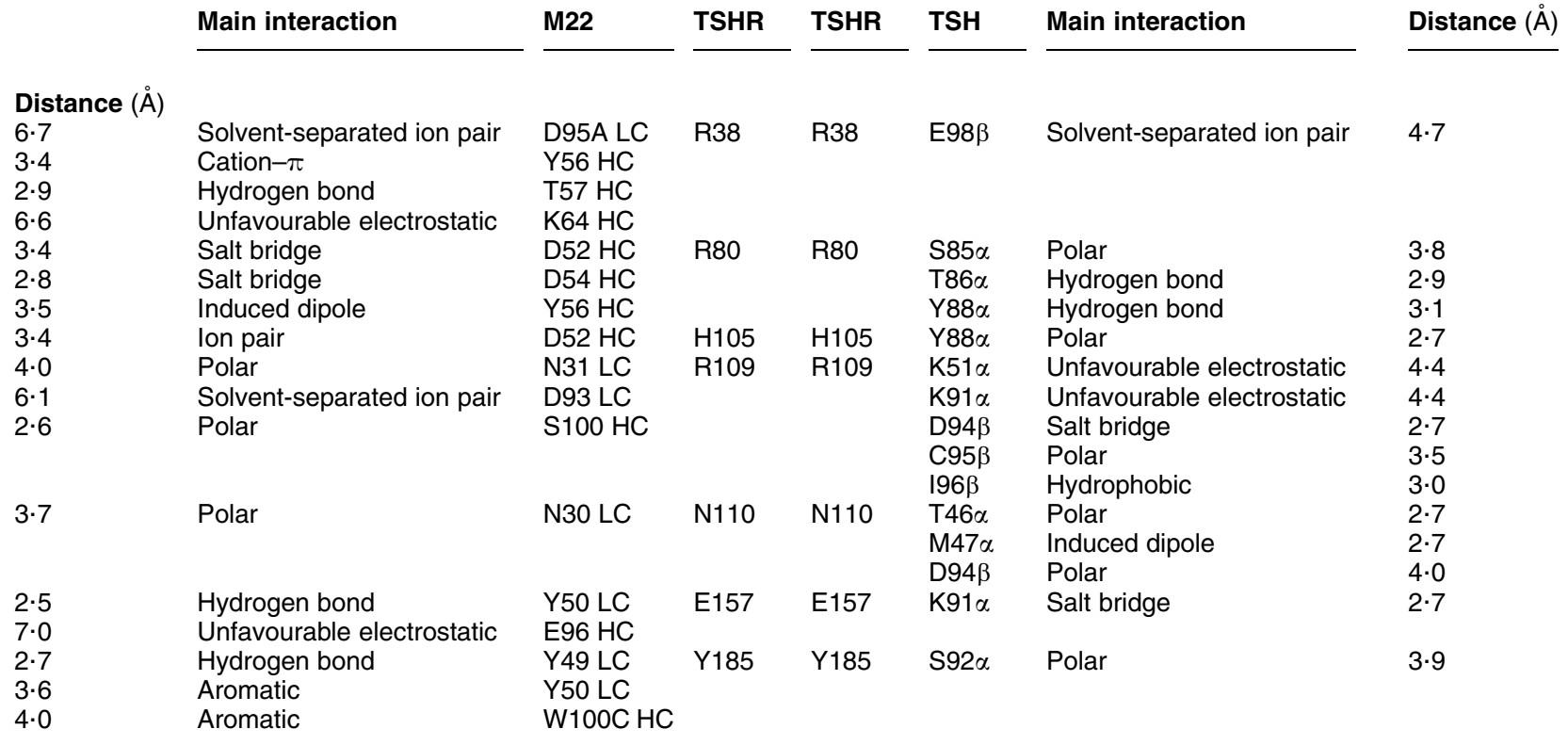

Ion-dipole and dipole-dipole interactions not involved in hydrogen bonds are described as 'polar'. Ion-induced dipole and dipole-induced dipole interactions are described as 'induced dipole'. Dispersion and hydrophobic forces are described as 'hydrophobic'. Charge-charge interactions at distance $\leq 3.5 \AA$ are defined as 'salt bridges'. Charge-charge interactions at distance $>3.5$ and $\leq 4.5 \AA$ are defined as 'ion pairs'. Charge-charge interactions at $>4.5 \AA$ distance are defined as 'solvent-separated ion pairs'. For explanation of cation- $\pi$ interactions see Gallivan \& Dougherty (1999).

the aromatic ring of $\mathrm{TSH} \alpha \mathrm{Y} 89$ in the respective complexes (Fig. 4A). TSHR K183A mutation caused an increase of binding affinity for porcine TSH (Sanders et al. 2006) and five to seven-fold increase of sensitivity to recombinant human TSH in a bioassay (Smits et al. 2003) but not an increase in porcine TSH induced cyclic AMP production compared with the wild-type TSHR (Sanders et al. 2006). By contrast, the TSHR K183D (change of charge) mutation showed a slight decrease in porcine TSH stimulated cyclic AMP production (Sanders et al. 2006). It has been proposed that TSHR residues K183 and E157 form an intramolecular salt bridge (Smits et al. 2002). Formation of this salt bridge is likely to reduce the strong interaction between TSHR E157 and TSH $\alpha$ K91 by reducing the charge of TSHR E157. In the mutation experiments, TSHR E157A substitution caused reduction of cyclic AMP response to stimulation with porcine $\mathrm{TSH}$ (60-40\% relative to wild-type). Consequently, substitutions at TSHR K183 are likely to increase TSH binding by breaking the intramolecular salt bridge and releasing TSHR E157 for strong binding with TSH. The strong reduction in M22 stimulated cyclic AMP production observed with the TSHR K183A mutant is probably due to the disruption of the salt bridge between TSHR K183 and M22 HC E96 (Fig. 4A). This is also consistent with the effect of M22 mutation HC
E96A which caused slight reduction of M22 stimulating activity (Sanders et al. 2007b).

Similar charge-charge interactions are observed in the respective complexes between TSHR K209 and the ligand residues M22 LC D52 and TSH $\beta$ D91. All residues are exposed to the solvent leading to reduction of the strength of the interactions (Table 4, Fig. 4B). Reduction in porcine TSH and M22 stimulated cyclic AMP production have been observed for the TSHR mutants K209A and K209E, however, the effect of these mutations on M22 biological activity was greater than on TSH activity (Smits et al. 2003, Sanders et al. 2006, $2007 a$ ). Furthermore, mutation M22 LC D52A resulted in the reduction of M22 stimulating activity $(<80-60 \%$ relative to the wild-type; Sanders et al. 2007b). TSHR K209 substitution to alanine would disrupt the chargecharge interactions in both complexes and substitution to glutamate is likely to produce a similar effect and also unfavourable interactions with both ligands.

\section{Differences in interactions in the two complexes}

Effects of different mutations in the TSHR and/or M22 on TSH and M22 binding and activity reported to date (Smits et al. 2003, Sanders et al. 2006, 2007a) can be explained by the interactions found in the M22-TSHR and TSH-TSHR complexes and Table 5 shows those 
Table 6 Comparison of interactions in the M22-TSHR and TSH-TSHR complexes. Interaction produced in the M22-TSHR complex only

\begin{tabular}{|c|c|c|}
\hline & $\begin{array}{l}\text { M22 } \\
\text { residue }\end{array}$ & Main interaction \\
\hline \multicolumn{3}{|l|}{ TSHR residue } \\
\hline E34 & D95A LC & $\begin{array}{l}\text { Unfavourable } \\
\text { electrostatic }\end{array}$ \\
\hline E35 & K64 HC & Ion pair \\
\hline D36 & $\mathrm{K} 64 \mathrm{HC}$ & $\begin{array}{l}\text { Solvent-separated } \\
\text { ion pair }\end{array}$ \\
\hline T56 & Y56 HC & Hydrophobic \\
\hline Y82 & Y99 HC & Aromatic \\
\hline \multirow[t]{2}{*}{ E107 } & G98 HC & Induced dipole \\
\hline & Y99 HC & Hydrogen bond \\
\hline \multirow[t]{4}{*}{ K129 } & $\mathrm{R} 28 \mathrm{HC}$ & $\begin{array}{l}\text { Unfavourable } \\
\text { electrostatic }\end{array}$ \\
\hline & T30 HC & 2 Hydrogen bonds \\
\hline & D52 HC & $\begin{array}{l}\text { Solvent-separated } \\
\text { ion pair }\end{array}$ \\
\hline & T53 HC & Hydrogen bond \\
\hline \multirow[t]{2}{*}{ N208 } & Y49 LC & Polar \\
\hline & Q53 LC & Hydrogen bond \\
\hline \multirow[t]{2}{*}{ Q235 } & D52 LC & Hydrogen bond \\
\hline & Q53 LC & Hydrogen bond \\
\hline \multirow[t]{5}{*}{ R255 } & L54 LC & Induced dipole \\
\hline & P55 LC & Polar \\
\hline & V58 LC & 2 Hydrogen bonds \\
\hline & S59 LC & Induced dipole \\
\hline & D60 LC & Ion pair \\
\hline N256 & L54 LC & Induced dipole \\
\hline
\end{tabular}

*No electron density was observed for the negatively charged oxygens of TSHR E35 in the M22-TSHR complex (Sanders et al. 2007a). Ion-dipole and dipole-dipole interactions not involved in hydrogen bonds are described as 'polar'. Ion-induced dipole and dipole-induced dipole interactions are described as 'induced dipole'. Dispersion and hydrophobic forces are described as 'hydrophobic'. Charge-charge interactions at distance $\leq 3.5 \AA$ are defined as 'salt bridges'. Charge-charge interactions at distance $>3.5$ and $\leq 4.5 \AA$ are defined as 'ion pairs'. Charge-charge interactions at $>4.5 \AA$ distance are defined as 'solvent-separated ion pairs'.

TSHR residues that produce different interactions with the two ligands.

For example, TSHR R109 shows some interactions with M22, two polar interactions, and one solventseparated ion pair (Table 5). The polar interaction with M22 HC S100 at $2 \cdot 6 \AA$ is not a hydrogen bond due to the incorrect angle. In the case of TSHR R109A mutation, these interactions would be absent and indeed decrease of M22 stimulating activity (40-60\% of the wild-type) associated with this mutation was observed (Sanders et al. 2006). TSHR R109D mutation would introduce an additional unfavourable electrostatic interaction with M22 LC D93 and this could explain reduced cyclic-AMP response to M22 stimulation to $<20 \%$ of the wild-type (Sanders et al. 2006). Although TSHR R109 has several interactions with TSH, including a strong salt bridge, substitutions at TSHR R109 have much smaller effect on TSH action. In the case of TSHR R109A mutation, responsiveness to
TSH stimulation is similar to the wild-type, however, TSH binding is markedly reduced (Sanders et al. 2006) consistent with the loss of interactions in the mutant. Furthermore, in the TSHR R109D mutant, stimulation by TSH is only $60-80 \%$ of the wild-type (Sanders $e t$ al. 2006). In this mutant, the salt bridge would change for an unfavourable electrostatic interaction, while the two unfavourable interactions in the wild-type receptor (Table 5) would change for two favourable interactions.

Only the case of TSHR R80 (Table 5) needs some clarification. TSHR R80 produces two hydrogen bonds and one polar interaction with the $\alpha$-chain of TSH. However, R80 also shows a strong intramolecuar hydrogen bond with TSHR Y82 and two intramolecular polar interactions with TSHR H105. These intramolecular interactions are likely to reduce the extent of the intermolecular hydrogen bonds. In mutation experiments, TSHR R80A showed similar porcine TSH binding and cyclic AMP response to porcine TSH as the wild-type TSHR while change of charge mutation (TSHR R80D) caused a small reduction in response to stimulation by porcine TSH $(80-100 \%$ of the wildtype; Sanders et al. 2006) most likely due to structure modification. By contrast, TSHR mutations R80A and R80D had marked effects on the ability of the TSHR to respond to stimulation by M22 $(<20 \%$ relative to the wild-type; Sanders et al. 2006). In the M22-TSHR complex, TSHR R80 forms two salt bridges with M22 HC D52 and M22 HC D54. M22 HC D52A, D52K, and D54R mutants showed $40-60 \%,<20 \%$ and $20-40 \%$, cyclic-AMP activity relative to the M22 wild-type respectively (Sanders et al. 2007b). This indicates that the two salt bridges are important for interactions between M22 and the TSHR in the complex.

There is an aromatic patch in the interface of the M22-TSHR complex between TSHR Y185 and two tyrosines and one tryptophan from M22 (Table 5). M22 HC W100C and M22 LC Y49 are in a face to face aromatic interaction. M22LC Y50 is in face to side interaction with two rings, M22HC W100C and M22LC Y49. Finally, TSHR Y185 has a face to side aromatic interaction with M22 LC Y50 and a hydrogen bond with M22LC Y49. This aromatic patch is likely to play an important role in the stabilization of the complex and this is consistent with TSHR Y185A causing considerable reduction in M22 binding affinity and stimulating activity (20-40\% of the wild-type; Sanders et al. 2006).

\section{TSHR residues that interact with one of the ligands only}

The analysis of interactions in the M22-TSHR and TSH-TSHR complexes has identified several TSHR residues that interact with M22 but not with TSH and these are shown in Table 6. Effects of mutations of M22 residues listed in Table 6 on M22 binding to the TSHR 

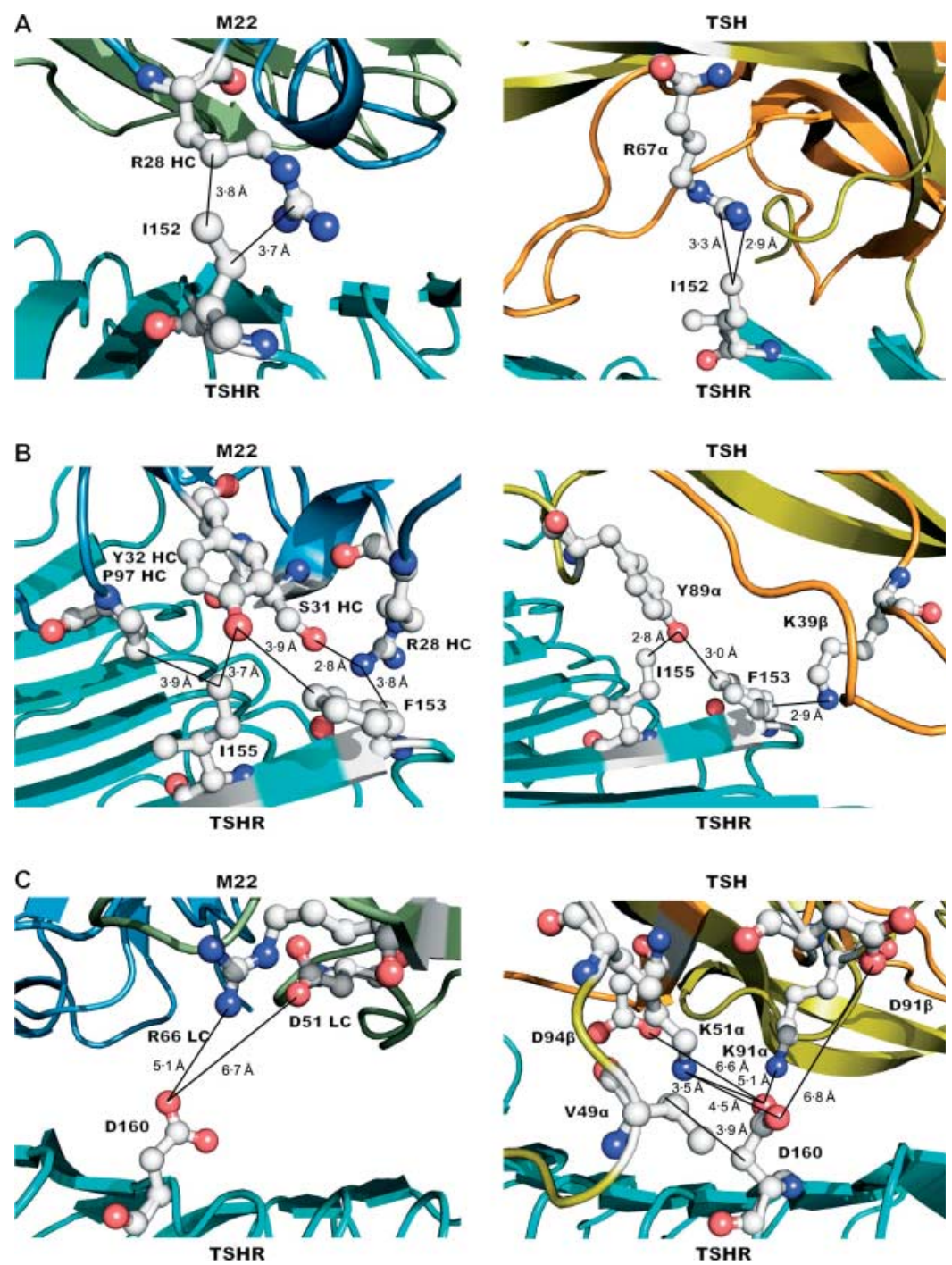

Figure 3 Interactions of (A) TSHR I152 with M22 heavy chain residue R28 and with TSH $\alpha$ R67, (B) TSHR F153 and I155 with M22 heavy chain residues and with TSH residues, and (C) TSHR D160 with M22 light chain residues and with TSH residues in the M22-TSHR and TSH-TSHR complexes. TSHR LRD backbones are shown in cyan, hormone $\alpha$-chains in olive and $\beta$-chains in orange, M22 light chain in green and heavy chain in blue. Interacting residues are shown as ball and sticks with carbon atoms in grey, oxygens in red, and nitrogens in blue. Solid lines denote interactions.

and TSHR cyclic AMP stimulation that have been reported previously (Sanders et al. 2007b) and can be explained by interactions observed in the M22-TSHR complex (Sanders et al. 2007a). In the case of the TSHR residues in Table 6 , mutation experiments have shown (Sanders et al. 2006) no significant changes in TSH binding or TSH mediated receptor activation except for TSHR E107.

TSHR E107 does not interact directly with TSH as the distances between TSHR E107 and TSH residues are too great for effective interactions (Fig. 4C). In addition, TSHR E107 is $7 \cdot 7 \AA$ away from TSH $\alpha$ K91 with no direct interaction (Fig. 4C). The E107A mutation caused a small reduction in stimulation by porcine TSH (60-80\% of the wild-type; Sanders et al. 2006) probably due to structure modification. The TSHR E107R mutation resulted in a more marked reduction in stimulation by porcine TSH $(20-40 \%$ of the wild-type; Sanders et al. 2006). In this TSHR E107R mutation, the longer side-chain of the new arginine is likely to be positioned closer to TSH $\alpha$ K91 resulting in an unfavourable charge-charge interaction. In the M22-TSHR complex, TSHR E107 is hydrogen bonded to the nitrogen of the backbone of M22 HC Y99 

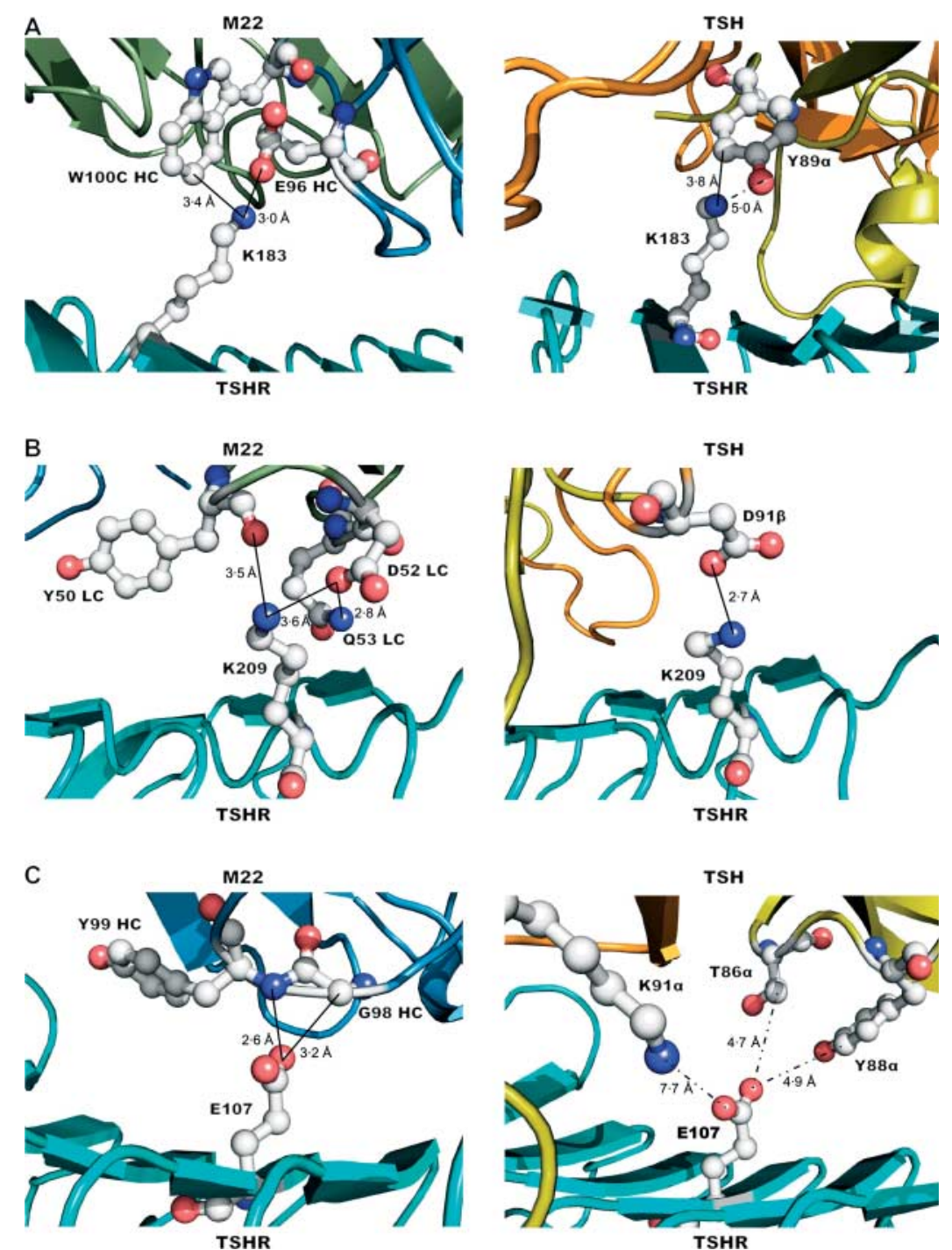

Figure 4 Interactions of (A) TSHR K183 with M22 heavy chain residues and with TSH $\alpha$ Y89, (B) TSHR K209 with M22 light chain residues and with TSH $\beta$ D91, and (C) TSHR E107 with M22 heavy chain residues and with $\mathrm{TSH} \alpha$ residues in the M22-TSHR and TSH-TSHR complexes. TSHR LRD backbones are shown in cyan, hormone $\alpha$-chains in olive and $\beta$-chains in orange, M22 light chain in green and heavy chain in blue. Interacting residues are shown as ball and sticks with carbon atoms in grey, oxygens in red, and nitrogens in blue. Solid lines denote interactions, dashed lines denote no interactions.

(Table 6, Fig. 4C). Substitutions at position E107 in TSHR cause a marked reduction in M22 stimulation ( $<20 \%$ relative to the wild-type; Sanders et al. 2006), probably due to the disruption of this hydrogen bond. Indeed, solvent inaccessible main chain $\mathrm{NH}$ groups are rarely found without a hydrogen bond partner at protein-protein interfaces or in protein cores (Baker \& Hubbard 1984).

TSHR R38 is hydrogen bonded to M22 HC T57 and shows a cation- $\pi$ interaction (Gallivan \& Dougherty 1999) with M22 HC Y56 (Table 5). The latter also shows some van der Waals interactions with TSHR (Tables 5 and 6). M22 HC Y56A variant shows reduction in stimulation of wild-type TSHR (40-60\% of the wildtype; Sanders et al. 2007b) consistent with the disruption of the cation $-\pi$ interaction.

TSHR Y82 has been previously identified as important for receptor specificity in discriminating between TSH and FSH (Núñez Miguel et al. 2008). The FSHR equivalent residue is in an ion pair interaction with FSH while TSHR Y82 does not interact with TSH (Núñez Miguel et al. 2008). However, TSHR Y82 is in an 
aromatic interaction with M22 HC Y99 (Table 6). The importance of this aromatic interaction is highlighted by the TSHR Y82A mutation associated with decrease of response to M22 stimulation (60-80\% of the wild-type; Sanders et al. 2006).

TSHR K129 is involved in strong interactions with M22 but does not interact with TSH. There are three hydrogen bonds and a solvent-separated ion pair between TSHR K129 and the M22 HC (Table 6). Substitutions at TSHR K129 caused marked reduction of cyclic-AMP production in response to M22 while similar levels of cyclic AMP were found in response to TSH. In the case of TSHR K129A mutant, the M22 stimulated cyclic-AMP accumulation was reduced to $40-60 \%$ of the wild-type (Sanders et al. 2006) while in the case of TSHR K129D it was reduced to $<20 \%$ of the wild-type (Sanders et al. 2006) indicating that the interactions involving TSHR K129 are important for M22 but not TSH.

TSHR R255 forms strong interactions with M22, a double hydrogen bond with M22 LC V58, one ion pair with M22 LC D60 and several van der Waals interactions (Table 6), while does not interact with TSH. TSHR R255A mutation caused a reduction in cyclic-AMP production in response to M22 stimulation $(40-60 \%$ of the wild-type; Sanders et al. 2006), in agreement with the disruption of the strong interactions between the mutant and M22. Furthermore, the TSHR R255D mutation caused a more marked reduction in M22 stimulated cyclic-AMP accumulation $(<20 \%$ relative to the wild-type; Sanders et al. 2006). In this case, in addition to the disruption of interaction, an unfavourable charge-charge interaction is introduced in the complex with the mutated TSHR.

TSHR residues that interact only with the native hormone TSH but not with M22 are shown in Table 7 . Out of the TSHR mutations reported to date involving the residues in Table 7 (Smits et al. 2003, Sanders et al. 2006), the only substitution in which a small reduction in M22 stimulation was observed corresponds to TSHR D203A (80-100\% of wild-type; Sanders et al. 2006). The reduction in activity is probably due to the modification of structure of the mutant as a similar reduction in cyclic-AMP production in response to TSH stimulation is also observed.

TSHR E251 forms a salt bridge with TSH $\beta$ K44 (Table 7). No reduction in response to TSH stimulation was detected with the TSHR E251A mutant (Sanders et al. 2006). This is probably because: 1 ) TSH $\beta$ K44 is involved in several interactions with the receptor and presumably, the disruption of one of these interactions is not enough to show a significant reduction in activation and 2) TSHR E251 and TSH $\beta$ K44 are exposed to the solvent reducing the strength of the interaction.
Table 7 Comparison of interactions in the M22-TSH receptor (TSHR) and TSH-TSHR complexes. Interaction produced in the TSH-TSHR complex only

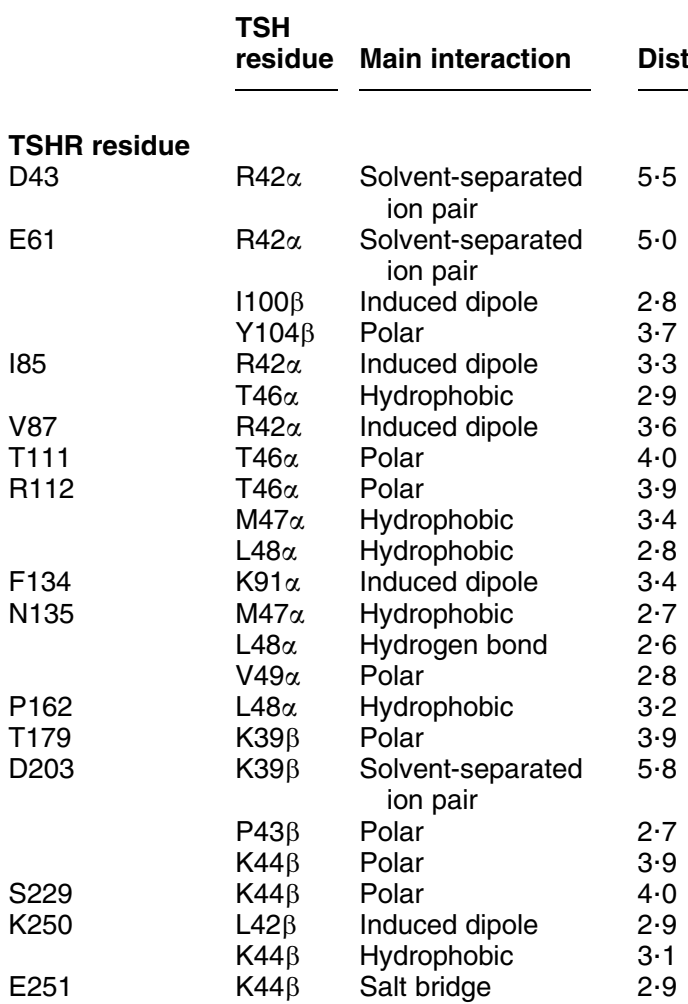

Ion-dipole and dipole-dipole interactions not involved in hydrogen bonds are described as 'polar'. Ion-induced dipole and dipole-induced dipole interactions are described as 'induced dipole'. Dispersion and hydrophobic forces are described as 'hydrophobic'. Charge-charge interactions at distance $\leq 3.5 \AA$ are defined as 'salt bridges'. Charge-charge interactions at distance $>3.5$ and $\leq 4.5 \AA$ are defined as 'ion pairs'. Charge-charge interactions at $>4.5 \AA$ distance are defined as 'solvent-separated ion pairs'.

The TSHR P162A variant has been reported to be associated with hypothyroidism (Sunthornthepvarakul et al. 1995, Costagliola et al. 1999). The maximal luciferase activity induced by recombinant human TSH was 20 times higher than the basal level in COS-7 cells transfected with wild-type TSHR. In order to produce a similar effect in cells transfected with TSHR P162A, 10 times more recombinant TSH was needed (Sunthornthepvarakul et al. 1995). Furthermore, in COS cells transfected with TSHR P162A, the $\mathrm{EC}_{50}$ was two-fold higher for cAMP accumulation in response to bovine TSH stimulation (Costagliola et al. 1999). The P162A mutation is likely to produce a disruption of the hydrophobic interaction with $\alpha \mathrm{TSH}$ L48 and a change in conformation produced by the phi angle restriction of prolines (around $-60^{\circ}$, phi is the torsion angle around the $\mathrm{N}^{-} \mathrm{C}_{\alpha}$ bond; Lovell et al. 2003). 


\section{Discussion}

Binding of M22 or TSH to the TSHR LRD is the first step in the process of the TSHR activation and studying these interactions is likely to provide insights into how autoantibodies cause TSHR activation.

It is remarkable that M22 LC interacts with the TSHR leucine rich repeats in a similar way to the $\beta$-chain of TSH (Table 1). Both, M22 LC and TSH $\beta$-chain interact more with the two ends of the concave face of the LRD than with the central part. M22 HC and TSH $\alpha$-chain also interact in a similar way with the TSHR leucine rich repeats and both interact mostly with the central part of the concave face of the LRD (Table 1). Furthermore, the results in Table 4 show that where similar interactions occur, M22 HC and TSH $\alpha$-chain residues usually interact with the same set of TSHR residues and M22 LC and TSH $\beta$-chain residues interact with the same set of TSHR residues. Each antibody chain appears to be mimicking one of the hormone chains in their interactions with the receptor. Furthermore, M22 and TSH have similar electrostatic surface potentials in the areas that interact with the TSHR LRD (Fig. 1B). There are two areas on the surface of M22 with similar charge distributions to two equivalent areas on the surface of TSH (see above). This is a clear case of molecular mimicry by the receptor autoantibody.

The M22-TSHR complex involves more strong interactions (eight salt bridges and 14 hydrogen bonds) and fewer hydrophobic interactions $(n=14)$ than the TSH-TSHR complex $(n=4, n=3$ and $n=22$ respectively; Table 2). The interaction between TSH and the TSHR is not of the highest affinity but this lower affinity may have evolved to optimize well controlled signal transduction, requiring both rapid receptor binding and rapid dissociation of the hormone. The mainly hydrophobic and polar interactions in the TSHTSHR complex facilitate this type of interaction. The interactions in the M22-TSHR complex rely more on strong interactions such as salt bridges and hydrogen bonds.

The interface $\triangle$ ASA is similar in both complexes, the $\triangle$ ASA value of the TSHR-TSH complex being only $19 \AA^{2}$ higher than that of the TSHR-M22 complex (Table 3). However, the gap volume of the TSHR-TSH complex is considerably higher than that of the TSHR-M22 complex, i.e. the gap volume index of the TSHR-TSH complex is $26 \%$ higher than that of the TSHR-M22 complex. This observation together with stronger interactions in the M22-TSHR complex is in agreement with the higher binding affinity of M22 for the TSHR compared with TSH found in experimental studies (Sanders et al. 2004, Rees Smith et al. 2007).

Consequently, the type of interactions observed and gap analysis in the crystal structure of the TSHR-M22 complex and in our model of the TSHR-TSH complex are consistent with the different binding affinities of the two ligands with the receptor. It may be, however, that M22 and TSH form interactions with parts of the TSHR in addition to the LRD (Sanders et al. 2007a,b, Mueller et al. 2008, Núñez Miguel et al. 2008). For example, different interaction of TSH of different species with non-LRD regions on the TSHR has been suggested to be important for differences in their binding affinities (Szkudlinski et al. 1996, Mizutori et al. 2008, Mueller et al. 2008, Núñez Miguel et al. 2008) but presently available crystal structures and comparative models provide little information regarding the relative positions of the various TSHR domains with respect to each other. Consequently, it is not yet possible to predict with confidence details of the interactions between M22 or TSH and these non-LRD domains.

The crystal structure of the M22-TSHR LRD complex shows the glycosylation sites on the TSHR LRD (N77, N99, N113, N177, and N198) and on the M22 (N26 LC) and these are located away from the concave surface of the LRD that interacts with M22 (Sanders $e t$ al. 2007a). Analysis of our model of the TSH-TSHR LRD complex shows that sugar residues from one component of the complex do not interact with sugar residues on the other component of the complex (Núnez Miguel et al. 2008). Also, human TSH glycosylation sites (TSH $\alpha$ N52 and N78 and TSH $\beta$ N23; Fig. 1A) are distant from the interacting interfaces and consequently are unlikely to be involved in interaction with receptor, at least between TSH and the TSHR LRD. This is similar to the situation observed in the FSH-FSHR LRD complex (Fan \& Hendrickson 2005). Some particular TSHR residues have been proposed previously to be important for specificity in terms of discriminating binding of TSH from other GPHs. For example, Smits et al. (2003) and Caltabiano et al. (2008) reported TSHR residues K58, E61, R80, Y82, I85, Y206, N208, and K209 to be important for this property. In the case of the FSHFSHR complex FSHR residues, L55, E76, R101, K179, and I222 (equivalent to TSHR residues E61, Y82, E107, N186 and L230) have been proposed to be important in hormone binding specificity (Fan \& Hendrickson 2005). In our studies on interactions in the FSHFSHR and TSH-TSHR complexes (Núñez Miguel et al. 2008), the TSHR residues: R38, T56, K58, R80, Y82, E107, K250, and E251 have been identified to be important for TSH binding specificity. Consequently, analyses carried out using different approaches showed that some of the TSHR residues reported to be important for specificity were the same in some studies but different in others (Smits et al. 2003, Fan \& Hendrickson 2005, Caltabiano et al. 2008, Núñez Miguel et al. 2008). For example, TSHR I85 that was proposed to be important for specificity in some studies (Smits $e t$ al. 2003, Caltabiano et al. 2008) in the TSH-TSHR model 
(Núñez Miguel et al. 2008) shows interactions with the $\alpha$-chain of TSH only and should therefore not be considered to be involved in specificity.

It is remarkable to observe that M22 interacts strongly with most of the TSHR residues predicted in different studies to be important for the specificity of TSH binding (Smits et al. 2003, Fan \& Hendrickson 2005, Caltabiano et al. 2008, Núñez Miguel et al. 2008) and strong interactions are observed between M22 and TSHR residues: R38, T56, K58, R80, Y82, E107, N208, and K209. Previous studies from our laboratory using competition between TSH and TSHR monoclonal antibodies have shown that TSHR amino acids 246-260 (as well as 277-296 and 381-385) are an important part of the TSH binding pocket (Jeffreys $e t$ al. 2002) and this is in agreement with the interactions seen in our TSH-TSHR model. Also a monoclonal antibody with weak thyroid stimulating activity has been shown to interact with TSHR amino acids 247-266 (Núñez Miguel et al. 2004). Furthermore, some of the TSHR residues important for interactions with TSH and M22 were found to be important for binding and/or stimulating activity of experimentally produced mouse or hamster TSHR monoclonal antibodies (Ando et al. 2002, Costagliola et al. 2004, Sanders et al. 2006).

The striking similarities in the interactions of M22 and TSH with the TSHR LRD we describe here are therefore supported by experimental evidence, but it is important to emphasize that our analysis of TSH-TSHR interactions is based on a comparative model rather than a true crystal structure. Also it should be noted that TSH forms interactions with parts of the TSHR outside the LRD as mentioned above.

The binding characteristics of M22 to the TSHR are representative of TSHR autoantibodies in sera from patients with Graves' disease (Sanders et al. 2004, 2006, Nakatake et al. 2006, Rees Smith et al. 2007). Furthermore, M22 and patient TSHR autoantibodies interact with the same region of the TSHR although there are some discrete differences in the actual amino acids on the concave surface of the TSHR LRD which contact different autoantibodies (Sanders et al. 2006). Identification of TSHR LRD residues that interact only with M22 but not TSH may be useful for developing means of inhibiting TSHR autoantibodies binding while retaining responsiveness of the receptor to TSH. As shown in Fig. 5, there are two areas on the concave surface of the TSHR LRD that interact with M22 but not with TSH. One of these, involving TSHR residues T56, Y82, E107, and K129, becomes buried upon TSH binding to the TSHR, although the interactions involved are weak. Consequently, blocking the accessibility of this patch for M22 will probably also block accessibility of TSH to the receptor. The second area involves TSHR residues N208, Q235, R255, and N256. This area is exposed in the TSH-TSHR complex

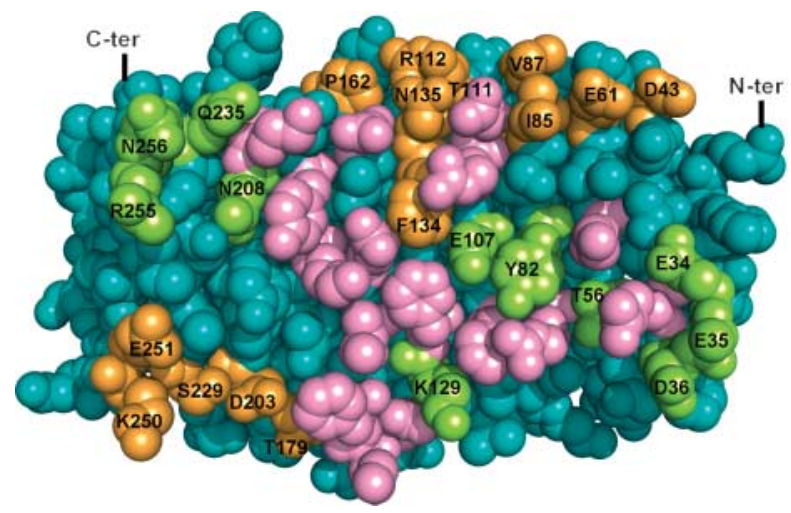

Figure 5 Space-filling representations of the TSHR LRD interactive surface: showing in green residues that interact only with $\mathrm{M} 22$, in orange residues that interact only with TSH and in pink those TSHR residues that interact with both ligands.

and is a good candidate for the development of small molecules which would bind to this patch in a way that would prevent autoantibody binding yet allow TSH to bind. Such small molecule inhibitors have the potential, at least hypothetically, to stop the biological activity of stimulating antibodies (Sanders et al. 2006, 2007a, Rees Smith et al. 2007). M22 interactions with the TSHR LRD observed in the M22-TSHR crystal structure are in good agreement with extensive experimental studies carried out with full-length TSHRs and therefore it would be expected that small molecules acting on the LRD would also inhibit antibody binding to intact TSHRs (Sanders et al. 2006, 2007a,b, Rees Smith et al. 2007). However, these molecules may or may not have an effect on binding and biological activity of at least some of TSHR autoantibodies with blocking activity (Rees Smith et al. 2007, Sanders et al. 2008b). Consequently, our results on M22 and TSH interactions with the TSHR suggest new possibilities to control ligand induced TSHR activity in Graves' disease.

In conclusion, our studies at the molecular level on the binding of two different ligands for the TSHR i.e. the thyroid stimulating autoantibody M22 and TSH show that the antibody LC mimics the binding of the hormone $\beta$-chain and the antibody $\mathrm{HC}$ mimics the binding of the hormone $\alpha$-chain. The higher TSHR binding affinity of M22 compared with TSH is explained by the interactions found in the complexes. An area on the TSHR surface has been identified which could be a suitable target for small molecules that could block autoantibody binding to the receptor while allowing hormone binding.

\section{Declaration of interest}

RSR Ltd is a developer of in vitro medical diagnostics including kits for measuring thyroid autoantibodies. RNM, JS, JF, and BRS are employees of RSR Ltd. 


\section{Funding}

The work was funded by RSR Ltd.

\section{Acknowledgements}

The authors would like to thank Professor Sir T L Blundell for his helpful discussions. Carol James prepared the manuscript.

\section{References}

Ando T, Latif R, Pritsker A, Moran T, Nagayama Y \& Davies TF 2002 A monoclonal thyroid-stimulating antibody. Journal of Clinical Investigation 110 1667-1674.

Baenziger JU \& Green ED 1988 Pituitary glycoprotein hormone oligosaccharides - structure, synthesis and function of the asparagine-linked oligosaccharides on lutropin, follitropin and thyrotropin. Biochimica et Biophysica Acta 947 287-306.

Baker EN \& Hubbard RE 1984 Hydrogen bonding in globular proteins. Progress in Biophysics and Molecular Biology 44 97-179.

Caltabiano G, Campillo M, De Leener A, Smits G, Vassart G, Costagliola S \& Pardo L 2008 The specificity of binding of glycoprotein hormones to their receptors. Cellular and Molecular Life Sciences 65 2484-2492.

Chazenbalk GD, McLachlan SM, Nagayama Y \& Rapoport B 1996 Is receptor cleavage into two subunits necessary for thyrotropin action? Biochemical and Biophysical Research Communications 225 $479-484$.

Cherezov V, Rosenbaum DM, Hanson MA, Rasmussen SGF, Thian FS, Kobilka TS, Choi H-J, Kuhn P, Weis WI, Kobilka BK et al. 2007 Highresolution crystal structure of an engineered human $\beta_{2}$-adrenergic G protein-coupled receptor. Science 318 1258-1265.

Claus M, Neumann S, Kleinau G, Krause G \& Paschke R 2006 Structural determinants for G-protein activation and specificity in the third intracellular loop of the thyroid-stimulating hormone receptor. Journal of Molecular Medicine 84 943-954.

Costagliola S, Sunthorntepvarakul T, Migeotte I, Van Sande J, Kajava AM, Refetoff S \& Vassart G 1999 Structure-function relationship of two loss-of-function mutations of the thyrotropin receptor gene. Thyroid 9 995-1000.

Costagliola S, Bonomi M, Morgenthaler NG, van Durme J, Panneels V, Refetoff S \& Vassart G 2004 Delineation of the discontinuousconformational epitope of a monoclonal antibody displaying full in vitro and in vivo thyrotropin activity. Molecular Endocrinology 18 3020-3034.

Fan QR \& Hendrickson WA 2005 Structure of human folliclestimulating hormone in complex with its receptor. Nature $\mathbf{4 3 3}$ 269-277.

Farid NR \& Szkudlinski MW 2004 Minireview: structural and functional evolution of the thyrotropin receptor. Endocrinology 145 4048-4057.

Felding-Habermann B, Lerner RA, Lillo A, Zhuang SF, Weber MR, Arrues S, Gao CS, Mao SL, Saven A \& Janda KD 2004 Combinatorial antibody libraries from cancer patients yield ligand-mimetic ArgGly-Asp-containing immunoglobulins that inhibit breast cancer metastasis. PNAS 101 17210-17215.

Foord SM, Bonner TI, Neubig RR, Rosser EM, Pin J-P, Davenport AP, Spedding M \& Harmar AJ 2005 International Union of Pharmacology. XLVI. G protein-coupled receptor list. Pharmacological Reviews 57 279-288.

Fox KM, Dias JA \& van Roey P 2001 Three-dimensional structure of human follicle-stimulating hormone. Molecular Endocrinology 15 378-389.
Fredriksson R, Lagerström MC, Lundin LG \& Schiöth HB 2003 The G-protein-coupled receptors in the human genome form five main families. Phylogenetic analysis, paralogon groups, and fingerprints. Molecular Pharmacology 63 1256-1272.

Gallivan JP \& Dougherty DA 1999 Cation- $\pi$ interactions in structural biology. PNAS 96 9459-9464.

Grossmann M, Szkudlinski MW, Tropea JE, Bishop LA, Thotakura NR, Schofield PR \& Weintraub BD 1995 Expression of human thyrotropin in cell lines with different glycosylation patterns combined with mutagenesis of specific glycosylation sites. Characterization of a novel role for the oligosaccharides in the in vitro and in vivo bioactivity. Journal of Biological Chemistry $\mathbf{2 7 0}$ 29378-29385.

Grossmann M, Weintraub BD \& Szkudlinski MW 1997 Novel insights into the molecular mechanisms of human thyrotropin action: structural, and therapeutic implications for the glycoprotein hormone family. Endocrine Reviews 18 476-501.

Jaakola V-P, Griffith MT, Hanson MA, Cherezov V, Chien EYT, Lane JR, Ijzerman AP \& Stevens RC 2008 The 2.6 Angstrom crystal structure of a human $\mathrm{A}_{2 \mathrm{~A}}$ adenosine receptor bound to an antagonist. Science 322 1211-1217.

Jeffreys J, Depraetere H, Sanders J, Oda Y, Evans M, Kiddie A, Richards T, Furmaniak J \& Rees Smith B 2002 Characterization of the thyrotropin binding pocket. Thyroid 12 1051-1061.

Jones S \& Thornton JM 1996 Principles of protein-protein interactions. PNAS 93 13-20.

Laskowski RA 1995 SURFNET: a program for visualizing molecular surfaces, cavities and intermolecular interactions. Journal of Molecular Graphics 13 323-330.

Lee B \& Richards FM 1971 The interpretation of protein structures: estimation of static accessibility. Journal of Molecular Biology $\mathbf{5 5}$ $379-400$.

Lovell SC, Davis IW, Arendall WB III, deBakker PIW, Word JM, Prisant MG, Richardson JS \& Richardson DC 2003 Structure validation by $\mathrm{C} \alpha$ geometry: $\Phi, \Psi$ and $\mathrm{C} \beta$ deviation. Proteins: Structure, Function, and Bioinformatics 50 437-450.

McDonald NQ \& Hendrickson WA 1993 A structural superfamily of growth factors containing a cystine knot motif. Cell 73 421-424.

McDonald IK \& Thornton JM 1994 Satisfying hydrogen bonding potential in proteins. Journal of Molecular Biology 238 777-793.

Mizutori Y, Chen C-R, McLachlan M \& Rapoport B 2008 The thyrotropin hinge region is not simply a scaffold for the leucine-rich domain but contributes to ligand binding and signal transduction. Molecular Endocrinology 22 1171-1182.

Mueller S, Kleinau G, Jaeschke H, Paschke R \& Krause G 2008 Extended hormone binding site of the human TSHR: distinctive acidic residues in the hinge region are involved in bovine TSH binding and receptor activation. Journal of Biological Chemistry 283 18048-18055.

Murakami M \& Kouyama T 2008 Crystal structure of squid rhodopsin. Nature 453 363-368.

Murray-Rust J, McDonald NQ, Blundell TL, Hosang M, Oefner C, Winkler F \& Bradshow RA 1993 Topogical similarities in TGF- $\beta 2$, PDGF-BB and NGF define a superfamily of polypeptide growth factors. Structure 1 153-159.

Nakatake N, Sanders J, Richards T, Burne P, Barrett C, Pra CD, Presotto F, Betterle C, Furmaniak J \& Smith BR 2006 Estimation of serum TSH receptor autoantibody concentration and affinity. Thyroid $\mathbf{1 6}$ 1077-1084.

Núñez Miguel R, Sanders J, Jeffreys J, Depraetere H, Blundell TL, Furmaniak J \& Rees Smith B 2003 TSH receptor cleavage domain and tissue inhibitor of metalloproteinase-2. Thyroid 13 665-666.

Núñez Miguel R, Sanders J, Jeffreys J, Depraetere H, Evans M, Richards T, Blundell TL, Rees Smith B \& Furmaniak J 2004 Analysis of the thyrotropin receptor-thyrotropin interaction by comparative modeling. Thyroid 14 991-1011. 
Núñez Miguel R, Sanders J, Chirgadze DY, Blundell TL, Furmaniak J \& Rees Smith B 2008 FSH and TSH binding to their respective receptors - similarities, differences and implication for glycoprotein hormone specificity. Journal of Molecular Endocrinology 41 145-164.

Palczewski K, Kamusaka T, Hori T, Behnke CA, Motoshima H, Fox BA, LeTrong I, Teller DC, Okada T, Stenkamp RE et al. 2000 Crystal structure of rhodopsin: a G protein-coupled receptor. Science $\mathbf{2 8 9}$ 739-745.

Rapoport B, Chazenbalk GD, Jaume JC \& McLachlan SM 1998 The thyrotropin (TSH) receptor: interaction with TSH and autoantibodies. Endocrine Reviews 19 673-716.

Rasmussen SGF, Choi HJ, Rosenbaum DM, Kobilka TS, Thian FS, Edwards PC, Burghammer M, Ratnala VRP, Sanishvili R, Fischetti RF et al. 2007 Crystal structure of the human beta(2) adrenergic G-protein-coupled receptor. Nature 450 383-388.

Rees Smith B, McLachlan SM \& Furmaniak J 1988 Autoantibodies to the thyrotropin receptor. Endocrine Reviews 9 106-121.

Rees Smith B, Sanders J \& Furmaniak J 2007 TSH receptor antibodies. Thyroid 17 923-938.

Richmond TJ 1984 Solvent accessible surface area and excluded volume in proteins. Analytical equations for overlapping spheres and implications for the hydrophobic effect. Journal of Molecular Biology 178 63-89.

Sanders J, Jeffreys J, Depraetere H, Richards T, Evans M, Kiddie A, Brereton K, Groenen M, Oda Y, Furmaniak JG et al. 2002 Thyroidstimulating monoclonal antibodies. Thyroid 12 1043-1050.

Sanders J, Evans M, Premawardhana LDKE, Depraetere H, Jeffreys J, Richards T, Furmaniak J \& Rees Smith B 2003 Human monoclonal thyroid stimulating autoantibody. Lancet 362 126-128.

Sanders J, Jeffreys J, Depraetere H, Evans M, Richards T, Kiddie A, Brereton K, Premawardhana LDKE, Chirgadze DY, Núñez Miguel R et al. 2004 Characteristics of a human monoclonal autoantibody to the thyrotropin receptor: sequence structure and function. Thyroid 14 560-570.

Sanders J, Bolton J, Sanders P, Jeffreys J, Nakatake N, Richards T, Evans M, Kiddie A, Summerhayes S, Roberts E et al. 2006 Effects of TSH receptor mutations on binding and biological activity of monoclonal antibodies and TSH. Thyroid 16 1195-1206.

Sanders J, Chirgadze DY, Sanders P, Baker S, Sullivan A, Bhardwaja A, Bolton J, Reeve M, Nakatake N, Evans M et al. 2007a Crystal structure of the TSH receptor in complex with a thyroid stimulating autoantibody. Thyroid 17 395-410.

Sanders J, Núñez Miguel R, Bolton J, Bhardwaja A, Sanders P, Nakatake N, Evans M, Blundell TL, Furmaniak J \& Rees Smith B $2007 b$ Molecular interactions between the TSH receptor and a thyroid stimulating monoclonal autoantibody. Thyroid 17 699-706.
Sanders J, Furmaniak J \& Rees Smith B $2008 a$ Crystal structure of the TSHR receptor. Patent publication number WO2008025991.

Sanders J, Evans M, Betterle C, Sanders P, Bhardwaja A, Young S, Roberts E, Wilmot J, Richards T, Kiddie A et al. 2008b A human monoclonal autoantibody to the thyrotropin receptor with thyroidstimulating blocking activity. Thyroid 18 735-746.

Scheerer P, Park JH, Hildebrand PW, Kim YJ, Krauß N, Choe H-W, Hofmann KP \& Ernst OP 2008 Crystal structure of opsin in its G-protein-interacting conformation. Nature 455 497-503.

Smith JW, Tudor Evans A, Costall B \& Smythe JW 2002 Thyroid hormones, brain function and cognition: a brief review. Neuroscience and Biobehavioral Reviews 26 45-60.

Smits G, Govaerts C, Nubourgh I, Pardo L, Vassart G \& Costagliola S 2002 Lysine 183 and glutamic acid 157 of the TSH receptor: two interacting residues with a key role in determining specificity toward TSH and human CG. Molecular Endocrinology 16 722-735.

Smits G, Campillo M, Govaerts C, Janssens V, Richter C, Vassart G, Pardo L \& Costagliola S 2003 Glycoprotein hormone receptors: determinants in leucine-rich repeats responsible for ligand specificity. EMBO Journal 22 2692-2703.

Sunthornthepvarakul T, Gottschalk ME, Hayashi Y \& Refetoff S 1995 Resistance to thyrotropin caused by mutations in the thyrotropinreceptor gene. New England Journal of Medicine 332 155-160.

Szkudlinski MW, Grossmann M \& Weitraub BD 1996 Structurefunction studies of human TSH. New advances in design of glycoprotein hormone analogs. Trends in Endocrinology and Metabolism 7 277-286.

Szkudlinski MW, Fremont V, Ronin C \& Weitraub BD 2002 Thyroidstimulating hormone and thyroid-stimulating hormone receptor structure-function relationship. Physiological Reviews 82 473-502.

Warne T, Serrano-Vega MJ, Baker JG, Moukhametzianov R, Edwards PC, Henderson R, Leslie AGW, Tate CG \& Schertler GFX 2008 Structure of a $\beta_{1}$ adrenergic G-protein-coupled receptor. Nature $\mathbf{4 5 4}$ $486-492$.

Wu H, Lustbader JW, Liu Y, Canfield RE \& Hendrickson WA 1994 Structure of human chorionic gonadotropin at 2.6 Angstroms resolution from MAD analysis of the selenomethionyl protein. Structure 2 545-558.

Wucherpfennig KW 2001 Structural basis of molecular mimicry. Journal of Autoimmunity 16 293-302.

Received in final form 3 February 2009

Accepted 16 February 2009

Made available online as an Accepted Preprint 16 February 2009 